\title{
Clinical implications of CT findings in mesenteric venous thrombosis at admission
}

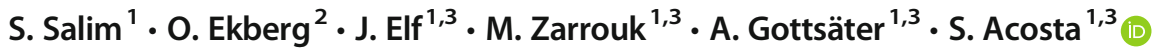

Received: 25 January 2018 / Accepted: 16 March 2018 / Published online: 28 March 2018

(C) The Author(s) 2018

\begin{abstract}
Purpose The main aim of this study was to evaluate the association of computed tomography (CT) findings at admission and bowel resection rate in patients with mesenteric venous thrombosis (MVT). It was hypothesized that abnormal intestinal findings on CT were associated with a higher bowel resection rate.

Methods Retrospective study of MVT patients treated between 2004 and 2017. CT images at admission and at follow-up were scrutinized according to a predefined protocol. Successful recanalization was defined as partial or complete recanalization of the portomesenteric venous thrombosis at the latest CT follow-up $(n=70)$.

Results We studied 102 patients (median age 58 years, 61 men). Lifelong anticoagulation was initiated in 64 patients, and bowel resection rate was $17 \%$. No referral letter indicated suspicion of MVT, whereas three indicated suspected intestinal ischemia. Previous venous thromboembolism was associated with increased bowel resection rate $(p=0.049)$. No patient with acute pancreatitis $(n=17)$ underwent bowel resection $(p=0.068)$. The presence of mesenteric oedema $(p=0.014)$, small bowel wall oedema $(p<0.001)$, small bowel dilatation $(p=0.005)$, and ascites $(p=0.021)$ were associated with increased bowel resection rate. Small bowel wall oedema remained as an independent risk factor associated with bowel resection (OR 15.8 [95\% CI 3.277.2]). Successful thrombus recanalization was achieved in $66 \%$ of patients.

Conclusion The presence of abnormal intestinal findings secondary to MVT confers an excess risk of need of bowel resection due to infarction. Responsible physicians should therefore scrutinize the CT images at diagnosis together with the radiologist to better tailor clinical surveillance.
\end{abstract}

Keywords Mesenteric venous thrombosis $\cdot \mathrm{CT} \cdot$ Intestinal ischemia $\cdot$ Bowel resection

\section{Introduction}

Mesenteric venous thrombosis (MVT) is an important cause of intestinal ischemia [1], though less common than mesenteric arterial thromboembolism. Unspecific abdominal pain is often present in the early stage of the disease, whereas localized

Electronic supplementary material The online version of this article (https://doi.org/10.1007/s10140-018-1601-3) contains supplementary material, which is available to authorized users.

S. Acosta

Stefan.acosta@med.lu.se

1 Department of Clinical Sciences, Lund University, Malmö, Sweden

2 Department of Translational Medicine, Division of Medical Radiology, Lund University, Malmö, Sweden

3 Vascular Centre, Department of Cardio-Thoracic and Vascular Surgery, Skåne University Hospital, SE-205 02 Malmö, Sweden abdominal pain develops later. Melena, hematemesis, or hematochezia occurs in only $15 \%$, whereas occult bleeding may be present in 50\% of the cases [2]. Fever and signs of peritonitis suggest progression of ischemia to intestinal infarction [3]. Since MVT is extremely seldom diagnosed on clinical grounds, computed tomography (CT) with intravenous contrast enhancement and imaging in the portal phase has become the most important, reliable, and accurate imaging for diagnosis of MVT [4-7]. CT can accurately visualize both the extent of thrombosis within the portomesenteric venous system and secondary abnormal intestinal findings. Thrombosis within the superior mesenteric vein is, in contrast to isolated portal vein thrombosis, associated with symptoms related to intestinal ischemia in the overwhelming majority (92\%) [8] and often results in intestinal infarction if left untreated $[8,9]$. Patients with MVT are nowadays, however, often diagnosed by CT in time, enabling a conservative medical approach with anticoagulation therapy [10]. 
One previous report suggests that patient characteristics and CT findings in MVT may be associated with increased bowel resection rate [11]. The main aim of the present large retrospective study was to evaluate the association of CT findings at admission and bowel resection due to intestinal infarction. It was hypothesized that abnormal intestinal findings on $\mathrm{CT}$ were associated with a higher bowel resection rate.

\section{Methods}

\section{Retrieval of patients with mesenteric venous thrombosis}

Ethical approval was obtained from the Regional Ethical Review Board in Lund (Dnr 2014/287). Identification of all patients with MVT treated surgically or conservatively at the Skåne University Hospital between 1st of January 2004 and 29th of September 2017 was performed in (1) hospital records based on the International Statistical Classification of Diseases and Related Health Problems (ICD), tenth edition, codes I81 (portal vein thrombosis [PVT] or MVT) and K55 (mesenteric ischemia), and (2) AuriculA [12] (a Swedish quality registry for patients treated with anticoagulation). All patient records and CT images in patients with PVT or MVT as well as unclear cases of mesenteric ischemia were scrutinized. Only patients with symptomatic thrombosis in the superior mesenteric vein with or without anatomical involvement of portal or splenic veins were included in the present study. Patients with isolated PVT without thrombotic involvement of the superior mesenteric vein were excluded. Median clinical follow-up was 48 months.

\section{Definitions}

Patients with abdominal pain of less than 4 weeks of duration were classified as having acute MVT. Those with symptoms for 4 weeks or more but without bowel infarction, and those with asymptomatic MVT diagnosed incidentally on abdominal imaging as a clinically non-significant finding were defined as chronic MVT. Extensive thrombosis was defined as having mesenteric central and peripheral, portal, and splenic vein thrombosis. The first five centimeters of the proximal superior mesenteric vein were defined as central. Small bowel dilatation was defined as $\geq 4 \mathrm{~cm}$ in bowel diameter. Patients initially treated with low molecular weight heparin (LMWH) for some weeks and later changed for VKA (vitamin K antagonists) or DOAC (direct-acting oral anticoagulants) were considered as treated with either of the respective oral anticoagulants.

Glomerular filtration rate (GFR) was calculated with a simplified variant of the Modification of Diet in Renal Disease Study Group (MDRD) equation [13], and advanced renal insufficiency was defined as GFR $<25 \mathrm{ml} / \mathrm{min}$.
Renal insufficiency evaluated by serum (s) creatinine alone was defined as a s-creatinine $>105 \mu \mathrm{mol} / \mathrm{l}(1.2 \mathrm{mg} / \mathrm{dl})$ in men and $>90 \mu \mathrm{mol} / \mathrm{l}(1.0 \mathrm{mg} / \mathrm{dl})$ in women. Body mass index (BMI) was defined as weight/length ${ }^{2}$ expressed in $\mathrm{kg} / \mathrm{m}^{2}$. The term "thrombophilia" was used as a common denominator for factors which might provoke MVT, such as cancer, coagulation disorders, previous or concomitant VTE, oral contraceptive use, or estrogen substitution. Inherited thrombophilic factors were defined as factor V Leiden mutation, prothrombin gene mutation, or deficiencies of protein $\mathrm{C}$, protein $\mathrm{S}$, or antithrombin. Acquired thrombophilic factors were defined as JAK2 V617F (janus-activated kinase gain of function substitute of valine to phenylalanine at position 617) mutation, lupus anticoagulant, or cardiolipin antibodies. A transient risk factor was defined as either recent surgery within 6 weeks, abdominal trauma, or inflammatory disease such as acute pancreatitis. Follow-up CT was defined as the last available CT of the abdomen with intravenous contrast and imaging in the parenchymal/venous phase. Successful recanalization was defined as partial or complete recanalization of the portomesenteric venous system at the follow-up CT after treatment.

\section{Computed tomography}

Clinical data provided in the referral letter for initial radiological examination at admission were retrieved in a Sectra radiological information system (Sectra AB, Linköping, Sweden). Multi-detector row CT (MDCT) was usually performed with a 0.75-mm slice thickness (Siemens Sensation 16, Erlangen, Germany). Multi-planar reconstruction in axial, coronal, and sagittal planes was usually obtained with a 5 -mm thickness. Single-slice CT was usually performed with slice thickness of $3-5 \mathrm{~mm}$. Patients were examined in the portomesenteric venous phase. Intravenous contrast medium was non-ionic contrast medium $300-320 \mathrm{mg} \mathrm{I} / \mathrm{ml}$ with a total dose of $90 \mathrm{ml}$, flow rate of $3 \mathrm{ml} / \mathrm{s}$, and delay of $70 \mathrm{~s}$. The diagnostic and follow-up CT scan images of all patients were scrutinized and evaluated by an experienced radiologist $(\mathrm{OE})$ aware of the diagnosis but blinded concerning which treatment the patient received. The main objective was to describe vascular and intestinal findings (Fig. 1) systematically in a predefined protocol [7]. Bowel wall thickness was assessed on non-contracted intestinal segments and defined as normal if $3 \mathrm{~mm}$ or less [14]. The patency of the portomesenteric venous system at follow-up CT was categorized as progression, unchanged, partial regression, and complete regression.

\section{Treatment strategy at the study center}

After diagnosis of MVT with CT, the mainstay of treatment was conservative with immediate full anticoagulation with either intravenous heparin infusion or subcutaneous LMWH, 


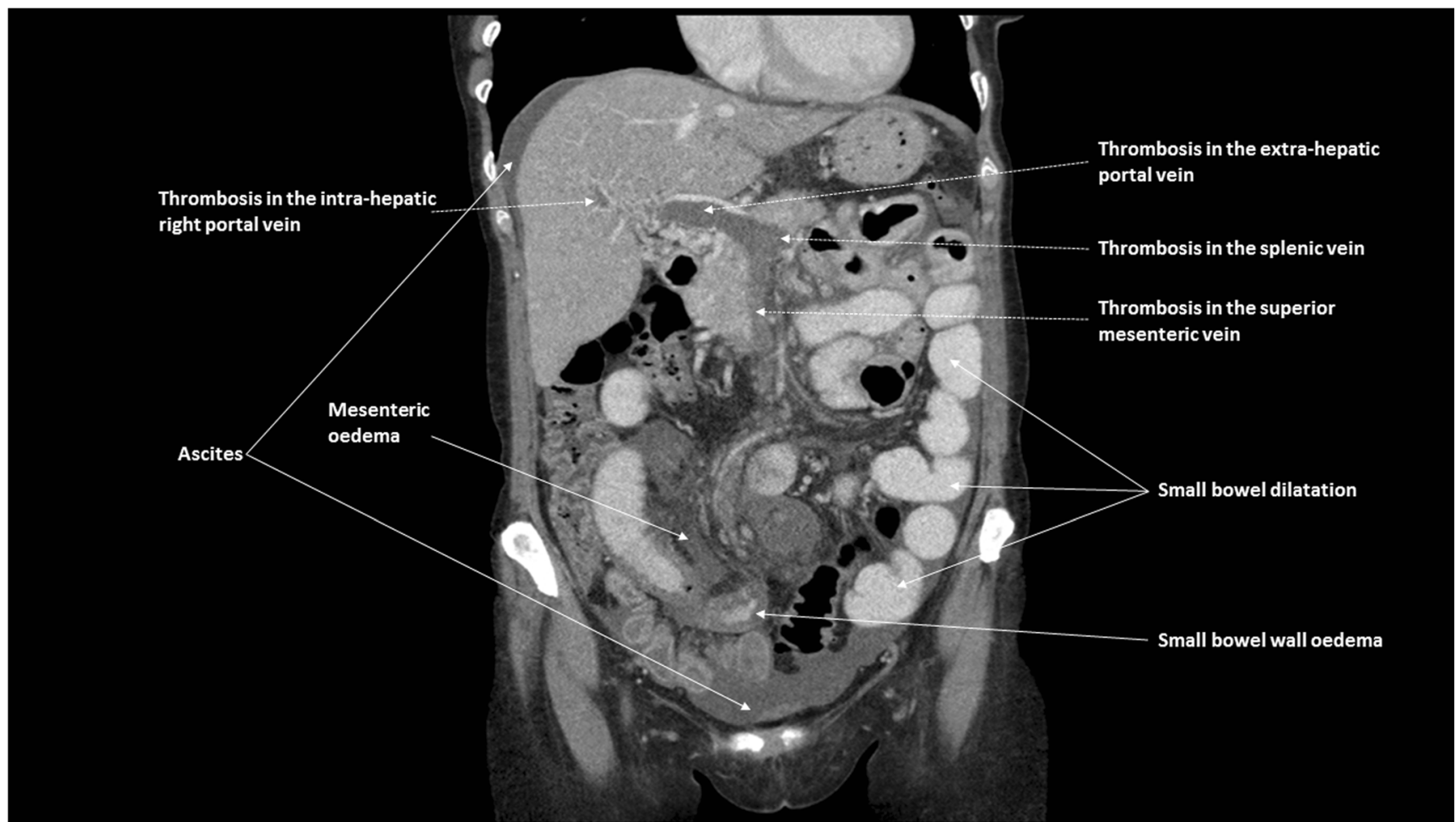

Fig. 1 A 59-year-old woman admitted with 3 days of abdominal pain and vomitus. CT with intravenous and oral positive contrast enhancement showed extensive portomesenteric venous thrombosis with secondary abnormal intestinal findings

full bowel rest, total parenteral nutrition, and analgesia. Patients admitted with peritonitis or rapidly progressing towards peritonitis underwent laparotomy and bowel resection. Patients not responding to anticoagulation may have undergone endovascular treatment with or without local thrombolysis, and non-responders were subjected to laparotomy. Clearly necrotic and demarcated bowels were resected and anastomosed. Bowels with unclear viability were usually evaluated at a second look laparotomy, and bowel resections were followed by reconstructions with anastomoses or diverting stomas. Patients with identified transient risk factors were usually treated with oral anticoagulation for 6 months, whereas those with permanent risk factors or unidentified risk factors were prescribed lifelong anticoagulation. Patients with a malignancy were usually treated with LMWH. DOACs were introduced for treatment of MVT at the study center in 2015.

\section{Statistical methods}

Data management and statistical analysis were performed using the SPSS for Windows program package (SPSS version 22.0, Chicago, IL, USA) or GraphPad (GraphPad Software, Inc., La Jolla, CA, USA). Distribution of variables was expressed with median value and interquartile range (IQR). Differences in proportions were evaluated using the chisquare or Fisher's exact test. Quantitative differences between groups were assessed with the Mann-Whitney $U$ test. When risk factor evaluation for bowel resection was performed, factors with $p<0.1$ in the uni-variable analysis were entered in a multi-variable regression analysis and expressed in odds ratio (OR) with 95\% confidence interval (CI). The Spearman rank test was used for calculating correlations. A $p$ value $<0.05$ was considered significant.

\section{Results}

\section{Patient characteristics}

During the 14-year period, 102 patients (61 men and 41 women) were diagnosed with MVT. Median age was 58 years (IQR, 47-68), and men (56 [IQR 47-64] years) were younger $(p=0.009)$ than women (65 [IQR 50-72] years). MVT was defined as acute in $100(98 \%)$ patients and chronic in the remaining 2 (2\%). Median BMI was 27.8 (IQR 25.5-31.4) in men $(n=49)$ and 25.5 (IQR 23.5-33.6) in women $(n=$ 34). One $(1.0 \% ; 1 / 100)$ patient had advanced renal insufficiency. Seventeen $(17 \%)$ patients had previously been diagnosed with pancreatitis, and 26 (26\%) with malignancies. Among 85 patients tested for thrombophilia, $17(20 \%)$ had the factor V Leiden mutation and 9 (9\%) had the JAK-2 V617 mutation. In 10 patients with myeloproliferative disease, 9 (90\%) were JAK-2 V617 mutation positive. Lifelong anticoagulation 
was initiated in 64 patients $(63 \%)$. Fifty-six $(55 \%)$ patients received VKA, 22 (22\%) LMWH, and 22 (22\%) DOAC.

\section{Clinical data from the referral letter for initial radiological examination}

Among the 102 patients with MVT, initial radiological examinations had been performed by CT in $69(68 \%)$, ultrasound in $26(26 \%)$, plain abdominal X-ray in $5(5 \%)$, and magnetic resonance imaging in $2(2 \%)$. None of the referral letters for initial radiological examination revealed any suspicion of MVT, whereas intestinal ischemia was suspected in $3(3 \%)$ patients. In these 3 patients with suspected intestinal ischemia, intestinal ischemia was mentioned among two, three, or four diagnostic suggestions in the referral letter. The spectrum of suspected clinical diagnoses at initial radiological examinations, respectively, is shown in Supplementary Table 1. The most frequently asked questions concerned intestinal disorders $(n=77)$, inflammatory disorders $(n=65)$, biliary or urinary tract disorders $(n=38)$, malignancies $(n=26)$, and benign disorders of the liver or spleen $(n=10)$.

\section{Vascular and intestinal CT findings}

Central and peripheral MVTs were documented in 98 (96\%) and $73(72 \%)$ patients, respectively. Extensive thrombosis at diagnostic CT was found in $43(42 \%)$ patients, and intestinal findings in $66(65 \%)$. The most frequent extra-vascular abnormalities were mesenteric oedema $(n=63 ; 62 \%)$, ascites $(n=$ $52 ; 51 \%)$, small bowel wall oedema $(n=40 ; 39 \%)$, and local small bowel dilatation $(n=10 ; 10 \%)$ (Table 1). No abnormalities in the colon were found.

\section{Radiological outcome-thrombus recanalization}

CT was performed both at diagnosis and after medical treatment in 70 patients after a median follow-up of 6 (IQR 3-28) months. The overall evaluation showed no change in 20 patients, progression of thrombotic status within the portomesenteric venous system in 4 patients, partial regression in 27 patients, and total regression in 19 patients. Successful recanalization had been achieved in $66 \%$ of the 70 patients (Table 2), $71 \%$ of those treated with VKA and $69 \%$ of those treated with DOAC ( $p=0.88)$. When entering age, malignancies, and type of therapy (VKA or DOAC) in a multi-variable analysis, none of these variables were associated with successful recanalization. Neither was there any correlation between successful recanalization and the time lapse between diagnostic and follow-up CT $(r=0.067, p=0.58)$. No clinical variable was found to be associated with successful recanalization.
Table 1 Vascular and intestinal findings on computed tomography at diagnosis of mesenteric venous thrombosis (MVT) in 102 patients

\begin{tabular}{lc}
\hline & Frequency $(\%)$ \\
\hline Vascular findings & \\
Central MVT & $98(96.1)$ \\
Peripheral MVT & $73(71.6)$ \\
Isolated MVT & $14(13.7)$ \\
Portal vein thrombosis & $85(83.3)$ \\
Extra-hepatic portal venous thrombosis & $80(78.4)$ \\
Intra-hepatic portal venous thrombosis & $60(58.8)$ \\
Splenic vein thrombosis & $61(59.8)$ \\
Venous collaterals & $52(51)$ \\
Extensive thrombosis & \\
Intestinal findings & $43(42.2)$ \\
Mesenteric oedema & $66(64.7)$ \\
Small bowel wall oedema & $63(61.8)$ \\
Local small bowel dilatation & $40(39.2)$ \\
Extensive small bowel dilatation & $10(9.8)$ \\
Gas in the portomesenteric venous system & $2(2.0)$ \\
Ascites & $0(0.0)$ \\
\hline
\end{tabular}

${ }^{\mathrm{a}}$ Mesenteric central and peripheral, portal, and splenic vein thrombosis

\section{Factors associated with bowel resection}

Among the 102 patients, 17 (17\%) underwent bowel resection. Previous VTE was associated with increased bowel resection rate $(p=0.049)$. No patient with exclusive transient risk factor $(n=$ 15) underwent a bowel resection $(p=0.069)$. No patient with acute pancreatitis $(n=17)$ underwent bowel resection $(p=$ $0.068)$. The presence of any intestinal finding at CT ( $p=$ $0.026)$, mesenteric oedema $(p=0.014)$, small bowel wall oedema $(p<0.001)$, small bowel dilatation $(p=0.005)$, and ascites $(p=0.021)$ were associated with increased bowel resection rate (Table 3). After entering small bowel wall oedema, acute pancreatitis, and previous VTE in a multi-variable regression analysis, small bowel wall oedema remained an independent risk factor associated with bowel resection (OR 15.8 [95\% CI 3.2-77.2], $p=0.001$ ), and previous VTE tended to be a risk factor for bowel resection (OR 3.3 [95\% CI 0.9-12.6], $p=0.080$ ).

\section{Mortality}

The 30-day mortality was 7\% (7/102). Mortality at the end of follow-up was $20 \%(20 / 102)$.

\section{Discussion}

The present study showed that a number of abnormal intestinal CT findings secondary to MVT such as small bowel wall 
Table 2 Recanalization of portomesenteric venous thrombosis verified by computed tomography (CT) after follow-up in 70 patients with mesenteric venous thrombosis (MVT)

\begin{tabular}{ll}
\hline Initial CT findings & Frequency of partial or complete recanalization (\%) \\
\hline All patients & $46 / 70(65.7)$ \\
Vascular findings & $45 / 69(65.2)$ \\
Central MVT & $34 / 46(73.9)$ \\
Peripheral MVT & $35 / 57(61.4)$ \\
Portal vein thrombosis & $35 / 56(62.5)$ \\
Extra-hepatic portal venous thrombosis & $28 / 42(66.7)$ \\
Intra-hepatic portal venous thrombosis & $23 / 43(53.5)$ \\
Splenic vein thrombosis & $22 / 39(56.4)$ \\
Venous collaterals & $17 / 27(63.0)$ \\
Extensive thrombosis & $29 / 45(64.4)$ \\
Intestinal findings & $28 / 44(63.6)$ \\
Mesenteric oedema & $20 / 27(74.1)$ \\
Small bowel wall oedema & $5 / 7(71.4)$ \\
Small bowel dilatation & $25 / 36(69.4)$ \\
Ascites &
\end{tabular}

${ }^{a}$ Mesenteric central and peripheral, portal, and splenic vein thrombosis oedema, small bowel dilatation, mesenteric oedema, and ascites were all associated with increased bowel resection rate, whereof small bowel wall oedema is the strongest risk factor. It therefore seems to be of utmost importance that CT images at diagnosis are scrutinized by the responsible physician together with the radiologist to better individualize clinical surveillance. The study data suggest that patients with small bowel wall oedema need a more close clinical follow-up than patients without secondary findings, with repeated physical examinations, C-reactive protein (CRP), and temperature measurements. In concordance with the present study, a recent study of 66 patients with CT verified MVT reports that bowel wall oedema, contrast enhancement defects of the bowel wall, and ascites were all associated with bowel resection [11]. The extent of thrombosis, involving both the superior mesenteric

Table 3 Association between vascular and intestinal findings at initial computed tomography (CT) and later need for bowel resection in 102 patients with mesenteric venous thrombosis (MVT)

\begin{tabular}{llc}
\hline CT findings & Bowel resection & $p$ value \\
\hline All & $17 / 102(16.7)$ & \\
Extensive thrombosis $^{\mathrm{a}}$ & $10 / 43(23.2)$ & 0.13 \\
Isolated MVT $_{\text {Intestinal findings }}$ & $2 / 14(14.3)$ & 1.0 \\
Mesenteric oedema & $15 / 66(22.7)$ & 0.026 \\
Small bowel wall oedema & $15 / 63(23.8)$ & 0.014 \\
Small bowel dilatation & $15 / 40(37.5)$ & $<0.001$ \\
Ascites & $6 / 12(50.0)$ & 0.005 \\
\hline
\end{tabular}

${ }^{a}$ Mesenteric central and peripheral, portal, and splenic vein thrombosis and portal veins as opposed to isolated thrombosis of the superior mesenteric vein, was also found to be associated with bowel resection [11], whereas in the present study, there was no increase in bowel resection rate in patients with extensive MVT, defined as SMV, PV, and splenic vein thrombosis. This might, however, be attributed to a type 2 statistical error. The decreased bowel resection rate in patients with a transient risk factor may imply that these patients have a more benign course of the disease and may therefore justify a limited 6month time period of anticoagulation [11]. In agreement, none of the 15 patients with an exclusive transient risk factor or of the 17 patients with acute pancreatitis, a transient strong provocative trigger, in the present study required bowel resection. In addition, we report a trend towards previous VTE being associated with the need of bowel resection in adjusted analysis, strengthening the indication for lifelong anticoagulation therapy in these patients.

Complete or partial radiological thrombus recanalization at follow-up CT was $66 \%$ among all patients, without difference between those treated with DOAC and those on VKA. However, the present explorative study was not optimally designed to compare results of VKA and DOAC treatment in MVT. In a radiological report on patients with acute MVT, $80 \%$ showed signs of evolution towards chronic MVT such as vein stenosis or occlusion and development of collateral veins [15]. It was reported that patients with short, isolated central MVT in a wide vein had a better chance of complete radiologic recovery [16]. Long-term imaging sequelae of portal venous hypertension, defined as esophageal varices, portal vein cavernous transformation, splenomegaly, or hepatic atrophy, were reported in $50 \%$ of MVT patients, and these radiological 
findings were associated with lower thrombus recanalization rate and more extensive thrombotic disease at initial CT [16]. Among MVT patients with extensive portomesenteric thrombosis in the present study, $63 \%$ achieved successful recanalization, a figure comparable with the overall rate of successful recanalization. To date, there is no proof that a follow-up CT evaluating changes of the status of the portomesenteric venous system is necessary. Nevertheless, the result of such a repeated CT can aid longterm decision-making. In case of complete clot resolution and recanalization, discontinuation of anticoagulation may be considered, particularly in patients with transient risk factors [1].

The retrospective design is a limitation of the present study. As the diagnosis of MVT is difficult to establish clinically as shown by the wide spectrum of conditions asked in the referral letters for the initial radiological assessment, a prospective study is impossible to design before a CT has been performed. After confirmation of MVT diagnosis by $\mathrm{CT}$, the rarity of the disease necessitates multi-center design to enable collection of prospective high-quality data. Another study limitation was that follow-up CT was performed in only $69 \%$ of our patients, mainly attributable to the absence of clear treatment recommendations for MVT patients. In addition, CT protocols at diagnosis and follow-up were often different, due to the wide diversity of questions asked in the referral letter for initial CT. Some initial CT examinations were performed with oral contrast media, which was never used at the follow-up CT evaluating the extent of portomesenteric venous thrombosis. When $\mathrm{CT}$ follow-up was considered, there was no fixed time point between the initial and follow-up CT, which might have influenced the thrombotic status in the portomesenteric venous system. Furthermore, the changes in thrombotic status were assessed by a semi-quantitative ordinal scale between CT examinations. With a prospective study design, a more modern quantitative evaluation using computer software for automatical or semi-automatical measurements of differences in thrombus volume [17] might be possible. Furthermore, appropriate statistical adjustment for several confounding variables in multi-variable analysis was not possible in our study, despite a rather large cohort of patients with MVT.

\section{Conclusion}

Abnormal intestinal CT findings secondary to MVT are related to excess risk of bowel resection due to intestinal infarction. Responsible physicians should therefore scrutinize initial CT images together with the radiologist to better tailor clinical surveillance. Thrombus recanalization rate after anticoagulation therapy was acceptable.
Funding Scandinavian Research Foundation of Venous Diseases.

\section{Compliance with ethical standards}

Ethical approval was obtained from the Regional Ethical Review Board in Lund (Dnr 2014/287).

Conflict of interest The authors declare that they have no conflict of interest

Open Access This article is distributed under the terms of the Creative Commons Attribution 4.0 International License (http:// creativecommons.org/licenses/by/4.0/), which permits unrestricted use, distribution, and reproduction in any medium, provided you give appropriate credit to the original author(s) and the source, provide a link to the Creative Commons license, and indicate if changes were made.

\section{References}

1. Björck M, Koelemay M, Acosta S, Bastos Goncalves F, Kolbel T, Kolkmann JJ, Lees T, Lefevre JH, Menyhei G, Oderich G (2017) Editor's choice - management of the diseases of mesenteric arteries and veins: clinical practice guidelines of the European Society of Vascular Surgery (ESVS). Eur J Vasc Endovasc Surg 53:460-510

2. Kumar S, Sarr MG, Kamath PS (2001) Mesenteric venous thrombosis. New England J Med 345:1683-1688

3. Hamoud B, Singal AK, Kamath PS (2014) Mesenteric venous thrombosis. J Clin Exp Hepatol 4:257-263

4. Oliva IB, Davarpanah AH, Rybicki FJ, Desjardins B, Flamm SD, Francois CJ, Gerhard-Herman MD, Kalva SP, Ashraf Mansour M, Mohler ER 3rd, Schenker MP, Weiss C, Dill KE (2013) ACR Appropriateness Criteria ${ }^{\circledR}$ imaging of mesenteric ischaemia. Abdom Imaging 38:714-719

5. Rajesh S, Mukund A, Arora A (2015) Imaging diagnosis of splanchnic venous thrombosis. Gastroenterol Res Pract 2015: 101029

6. Wayne E, Ough M, Wu A, Liao J, Andresen KJ, Kuehn D, Wilkinson N (2010) Management algorithm for pneumatosis intestinalis and portal venous gas: treatment and outcome of 88 consecutive cases. J Gastrointest Surg 14:437-448

7. Acosta S, Alhadad A, Ekberg O (2009) Findings in multi-detector row CT with portal phase enhancement in patients with mesenteric venous thrombosis. Emerg Radiology 16:477-482

8. Amitrano L, Guardascione MA, Scaglione M, Pezzullo L, Sangiuliano N, Armellino MF, Manguso F, Margaglione M, Ames PR, Iannaccone L, Grandone E, Romano L, Balzano A (2007) Prognostic factors in noncirrhotic patients with splanchnic vein thromboses. Am J Gastroenterol 102:2464-2470

9. Acosta S (2010) Epidemiology of mesenteric vascular disease: clinical implications. Semin Vasc Surg 23:4-8

10. Hagspiel KD, Flors L, Hanley M, Norton PT (2015) Computed tomography angiography and magnetic resonance angiography imaging of the mesenteric vasculature. Tech Vasc Interv Radiol 18:2-13

11. Kim HK, Hwang D, Park S, Lee JM, Huh S (2017) Treatment outcomes and risk factors for bowel infarction in patients with acute superior mesenteric venous thrombosis. J Vasc Surg Venous Lymphat Disorder 5:638-646

12. Wieloch M, Själander A, Frykman V, Rosenqvist M, Eriksson N, Svensson PJ (2011) Anticoagulation control in Sweden: reports of time in therapeutic range, major bleeding, and thrombo-embolic 
complications from the national quality registry AuriculA. Eur Heart J 32:2282-2289

13. Grubb A, Nyman U, Björk J, Lindström V, Rippe B, Sterner G, Christensson A (2005) Simple cystatin C-based prediction equations for glomerular filtration rate compared with the modification of diet in renal disease prediction equation for adults and the Schwartz and the Counahan-Barratt prediction equations for children. Clin Chem 51:1420-1431

14. Maglinte DDT, Herlinger H (1989) Anatomy of the small intestine. In Clinical radiology of the small intestine. Ed. Herlinger $\mathrm{H}$ and Maglinte DDT. WB Saunders Philadelphia, p 10

15. Vietti Violi N, Fournier N, Duran R, Schmidt S, Bize P, Guiu B, Denys A (2014) Acute mesenteric vein thrombosis: factors associated with evolution to chronic mesenteric vein thrombosis. AJR Am J Roentgenol 203(1):54-61

16. Maldonado TS, Blumberg SN, Sheth SU, Perreault G, Sadek M, Berland T, Adelman MA, Rockman CB (2016) Mesenteric vein thrombosis can be safely treated with anticoagulation but is associated with significant sequelae of portal hypertension. J Vasc Surg Venous Lymphat Disord 4:400-406

17. Nagaoki Y, Aikata H, Daijyo K, Teraoka Y, Shinohara F, Nakamura Y, Hatouka M, Morio K, Nakahara T, Kawaoka T, Tsuge M, Hiramatsu A, Imamura M, Kawakami Y, Ochi H, Chayama K (2017) Efficacy and safety of edoxaban for treatment of portal vein thrombosis following danaparoid sodium in patients with liver cirrhosis. Hepatol Res 48:51-58. https://doi.org/10.1111/hepr.12895 